\title{
Editorial
}

\section{Perturbation Methods and Formal Modeling for Dynamic Systems}

\author{
Saeed Islam, ${ }^{1}$ Sher Afzal Khan, ${ }^{2}$ Gul Zaman, ${ }^{3}$ and II Hyo Jung ${ }^{4}$ \\ ${ }^{1}$ Department of Mathematics, Abdul Wali Khan University, Mardan, Pakistan \\ ${ }^{2}$ Faculty of Computing and Information Technology in Rabigh, King Abdulaziz University, Jeddah, Saudi Arabia \\ ${ }^{3}$ Department of Mathematics, University of Malakand, Lower Dir, Pakistan \\ ${ }^{4}$ Department of Mathematics, Pusan National University, Busan 609-735, Republic of Korea
}

Correspondence should be addressed to Saeed Islam; saeedislam@awkum.edu.pk

Received 1 March 2015; Accepted 1 March 2015

Copyright (C) 2015 Saeed Islam et al. This is an open access article distributed under the Creative Commons Attribution License, which permits unrestricted use, distribution, and reproduction in any medium, provided the original work is properly cited.

The nonlinear differential equations have been variously used by the researchers in science and engineering to model many real world problems. If the problems from various branches of science and engineering are persistently unresolved through differential equations, the approximate solutions may be obtained through perturbation or numerical methods [1]. However, the availability of high speed computers, advanced algorithm, and a number of sophisticated numerical techniques has enabled researchers to solve problems through differential equations of considerable complexity and nonlinearity. Nonetheless, failures to find exact analytical solutions to problems have reverted the researchers to various approximate analytical methods $[2,3]$.

Since the power series method fails to provide solutions to equations at irregular singular points and at infinity, the asymptomatic method, which is an off-shoot of the power series method, provides the necessary answers to the singularly perturbed equations at infinity as well. The perturbation technique involves the expression of the unknown function of the problem to be solved as a power series expansion and obtain the successive terms of the expansion carefully.

The idea behind the perturbation method is that the initiative is taken with a simplified form of the original problem, and the perturbations or corrections are added gradually so that the original problem correlates with the solution. The different perturbation parameters are adopted starting from the minimal value taken as zero. The perturbation method is supplemented by boundary-layer method, multiple-scale method, WKB method, and so forth [3].

In problems involving linear or nonlinear differential equations, a sequence of linear subproblems is devised comprised of a number of differential equations; these are successively solved to obtain solution to the original problem.

Researchers have developed nonperturbation methods as well in order to solve complicated subproblems involving perturbed parameters. Among these, Lyapunov's artificial parameter method and (sigma) expansion method and Adomian decomposition method are important. Another most efficient and nicely developed technique is the homotopy analysis method proposed by Liao, homotopy perturbation method developed by He, optimal homotopy asymptotic method and optimal homotopy perturbation methods introduced by Marinca, and Herisanu semianalytical techniques which are best suited for nonlinear ordinary and partial differential equations [4-7].

However, formal modelings are based on mathematical techniques and notations, for describing and analyzing properties of software systems $[8,9]$.

These mathematical techniques are typically based on discrete mathematics such as predicate logic, set theory, relations, functions, and graph theory. The precise mathematical notation eliminates the ambiguity, inconsistencies, and incompleteness from any software and hardware system. The aim of formal methods is to demonstrate the process of 
development of a system from requirement to coding. This approach identifies errors and oversights early in the design life cycle, which are then easy to remove, with consequent high quality and cost saving. This improves the informal form of the system but does not replace them. There are various types of formal modeling approaches which include CommUnity, Estelle, Esterel, Lotos, Petri Nets, RAISE, Z, VDM, and $\mathrm{VDM}++[10-12]$. We can divide formal specification into three components: syntax: grammatical rules to determine if sentences are well formed, semantics: rules for interpreting the sentences in a precise, meaningful way within the domain, and proof theory: rules for inferring useful information from the specification.

\author{
Saeed Islam \\ Sher Afzal Khan \\ Gul Zaman \\ Il Hyo Jung
}

\title{
References
}

[1] A. H. Nayfeh, Perturbation Methods, Wiley, New York, NY, USA, 1972.

[2] J. H. He, "A review on some new recently developed non linear analytical techniques," International Journal of Nonlinear Sciences and Numerical Simulation, vol. 1, no. 1, pp. 51-70, 2000.

[3] T. S. L. Radhika, T. K. V. Iyengar, and T. R. Rani, Approximate Analytical Methods for Solving Ordinary Differential Equations, CRC Press, Taylor \& Francis Group, Boca Raton, Fla, USA, 2014.

[4] A.-M. Wawaz, "A reliable modification of Adomian decomposition method," Applied Mathematics and Computation, vol. 102, no. 1, pp. 77-86, 1999.

[5] S. J. Liao, The proposed homotopy analysis technique for the solutionof nonlinear problems [Ph.D. thesis], Shanghai Jiao Tong University, Shanghai, China, 1992.

[6] J.-H. He, "Homotopy perturbation technique," Computer Methods in Applied Mechanics and Engineering, vol. 178, no. 3-4, pp. 257-262, 1999.

[7] V. Marica and N. Hersanu, "Application of optimal homotopy asymptoticmethod for solving nonlinear equations arising in heat transfer," International Journal of Heat and Mass Transfer, vol. 13, pp. 710-715, 2008.

[8] E. A. Strunk, X. Yin, and J. C. Knight, "Echo: a practical approach to formal verification," in Proceedings of the 10th International Workshop on Formal Methods for Industrial Critical Systems (FMICS '05), pp. 44-53, ACM, Lisbon, Portugal, September 2005.

[9] M. C. Edmund and J. M. Winggie, Formal Methods: State of the Art and Future Direction, Carnegie Mellon University, Pittsburgh, Pa, USA, 1996.

[10] S. Liu and R. Adams, "Limitations of formal methods and an approach to implement," Tech. Rep., Hiroshima City University, Hiroshima, Japan, 1995.

[11] J. Woodcock and J. Davies, Using Z: Specification, Refinement, and Proof, Prentice Hall, 1996.

[12] J. P. Hinchey, Bowen, and G. Michael, Ten Commandments of Formal Methods, PC World Russia, 1997. 


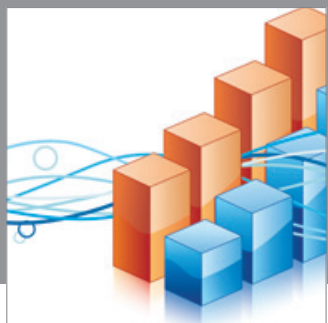

Advances in

Operations Research

mansans

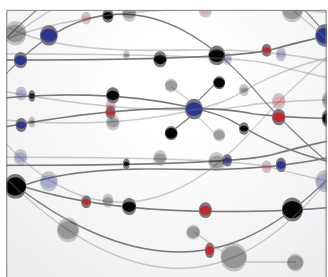

The Scientific World Journal
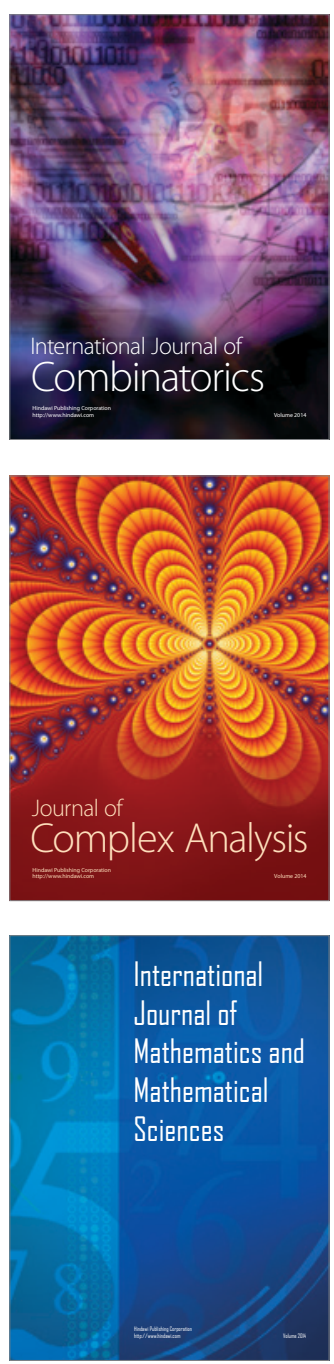
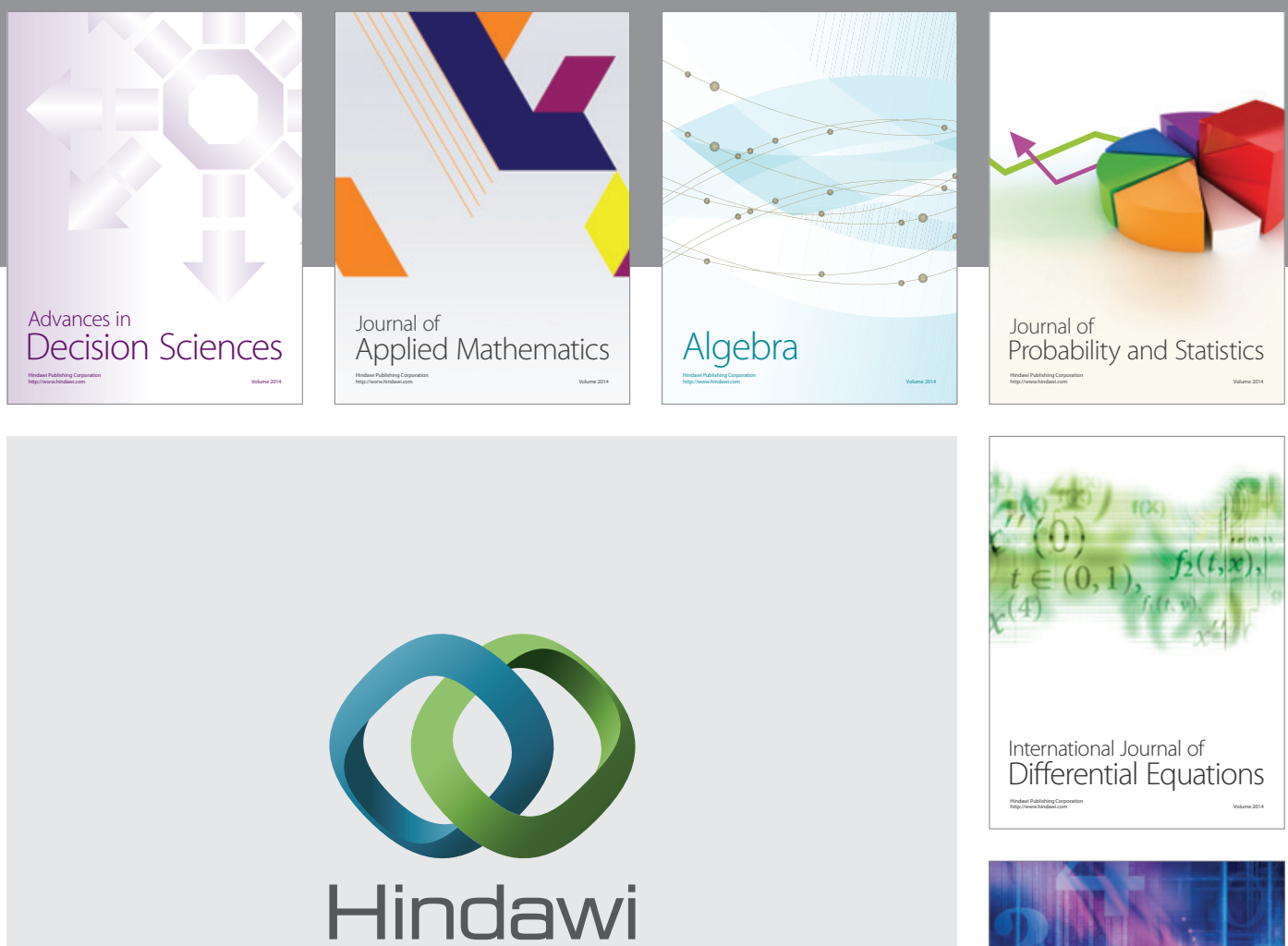

Submit your manuscripts at http://www.hindawi.com
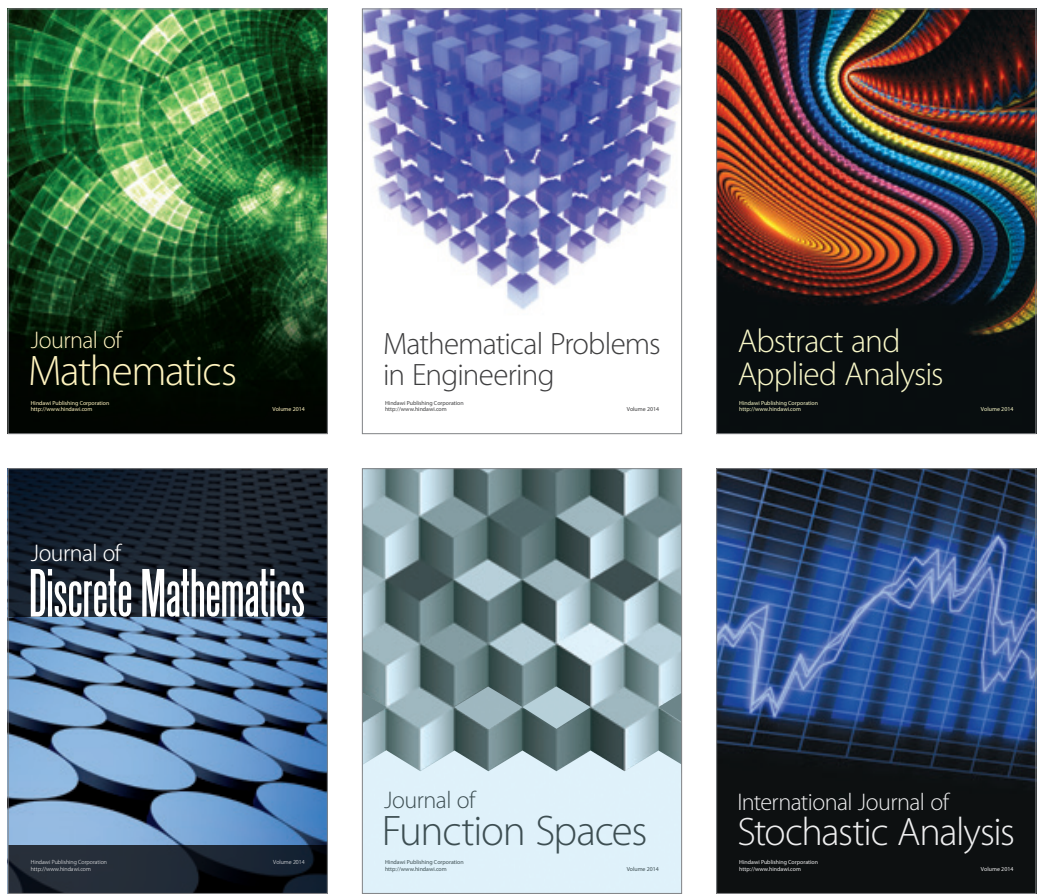

Journal of

Function Spaces

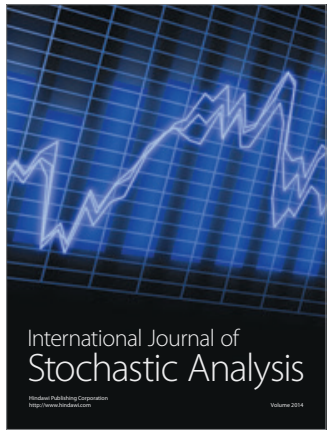

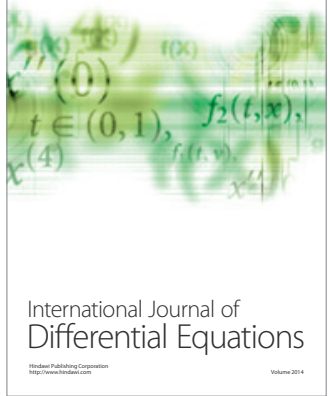
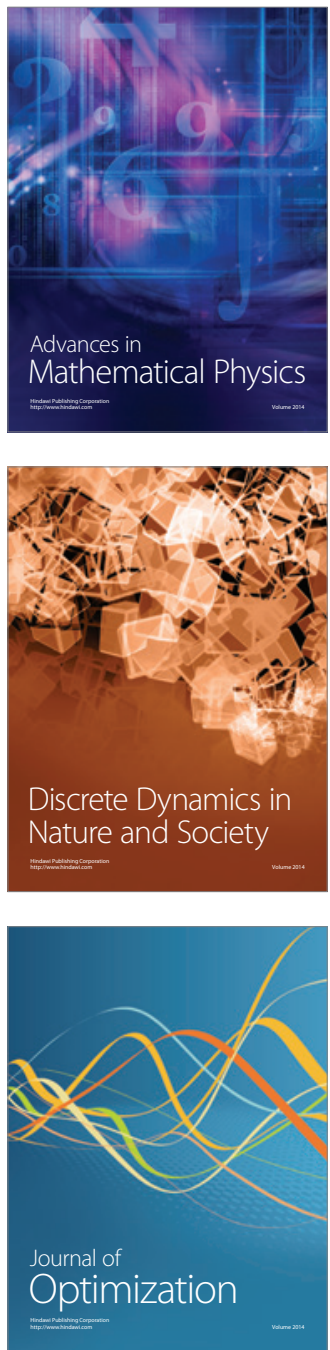